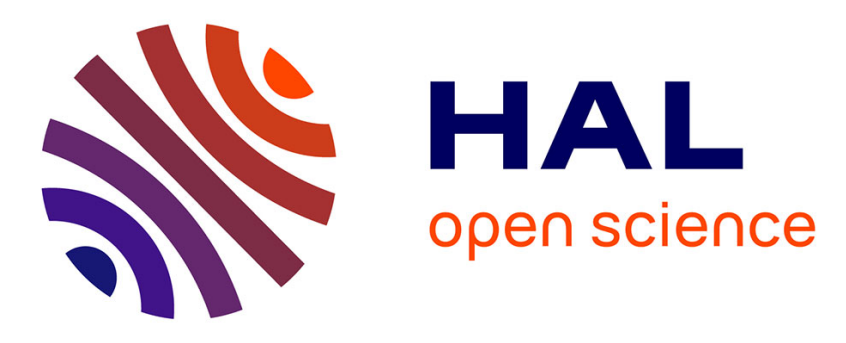

\title{
L'orchestration rituelle du partage des émotions et ses ressorts interactionnels
}

Marika Moisseeff, Michael Houseman

\section{To cite this version:}

Marika Moisseeff, Michael Houseman. L'orchestration rituelle du partage des émotions et ses ressorts interactionnels. Laurence Kaufmann; Louis Quéré. Émotions collectives. En quête d'un 'objet' impossible, 29, Éditions de l'École des Hautes Études en Sciences sociales, pp.133-168, 2020, Raisons pratiques. Epistémologie, sociologie, théorie sociale, 978-2-7132-2809-4. halshs-03096391

\section{HAL Id: halshs-03096391 https://shs.hal.science/halshs-03096391}

Submitted on 5 Jan 2021

HAL is a multi-disciplinary open access archive for the deposit and dissemination of scientific research documents, whether they are published or not. The documents may come from teaching and research institutions in France or abroad, or from public or private research centers.
L'archive ouverte pluridisciplinaire HAL, est destinée au dépôt et à la diffusion de documents scientifiques de niveau recherche, publiés ou non, émanant des établissements d'enseignement et de recherche français ou étrangers, des laboratoires publics ou privés. 
Publié en 2020 in Laurence Kaufmann et Louis Quéré (éds), Les émotions collectives. En quête d'un « objet » impossible, Paris, Éditions de l'EHESS, coll. 'Raisons pratiques', pp. 133-168.

\title{
L'ORCHESTRATION RITUELLE DU PARTAGE DES ÉMOTIONS ET SES RESSORTS INTERACTIONNELS
}

\author{
Marika Moisseeff (CNRS/Laboratoire d'anthropologie sociale, Université PSL) et Michael \\ Houseman (EPHE/Institut des mondes africains, Université PSL)
}

Les ethnologues n’ont cessé de s’interroger sur la spécificité des émotions particulièrement intenses qui se donnent à voir au cours de certaines activités collectives. A l'instar du travail fondateur d'Emile Durkheim en la matière (1912), ce questionnement se rapporte avant tout à des contextes rituels dont, en premier lieu, les pratiques funéraires (Mauss 1921 ; Malinowski 1927 ; Radcliffe-Brown 1922). En nous penchant à notre tour sur les émotions rituelles et sur le caractère "collectif » qu'il convient de leur reconnaitre, nous chercherons à souligner que, contrairement à ce que laissent entendre l'expression d' " effervescence » (Durkheim 1912) ou ses avatars récents tels qu'arousal (Whitehouse 2000) ou « énergie émotionnelle » (Collins 2004), les manifestations affectives caractéristiques des rites collectifs ne se résument nullement à un état d'excitation indifférenciée où, pour reprendre la façon de concevoir la foule de Gustav Le Bon, "l'hétérogène se noie dans l'homogène » $(1905,20)$. En effet, la structuration unitaire d'une pratique rituelle donnée, ce qui la rend reconnaissable (et transmissible) en tant que telle, repose typiquement sur la gestion continue d'une hétérogénéité à la fois comportementale et expressive. Pour développer cette idée, nous nous pencherons sur des rites relevant de diverses traditions culturelles afin d'illustrer trois aspects étroitement intriqués de la participation cérémonielle : (1) une volonté que partagent les participants de contribuer à une réalisation correcte du rituel, (2) une performance fondée sur la coordination systématique des disparités qui distinguent leurs comportements respectifs, (3) un accordage réciproque de l'intensité des expressions émotionnelles qu'implique la poursuite de ces comportements ${ }^{1}$.

Ceux qui prennent part à un rituel tel que des obsèques ou une célébration de mariage s'engagent sciemment dans une activité collective renvoyant à certains registres affectifs : la tristesse et la colère face à la perte d'un proche, la gaieté associée au consentement d'un amour réciproque. Toutefois, ce que partagent avant tout les acteurs est une même intention de contribuer au bon déroulement de la performance rituelle. Cette préoccupation est d'autant plus décisive que la collaboration qui se poursuit dans le cadre de cet alignement intentionnel passe typiquement par l'agencement de comportements distincts que doivent adopter différentes catégories de participants. Lors d'un mariage, par exemple, on ne s'attend certainement pas à ce que les témoins, les parents des mariés, les mariés eux-mêmes et les membres de l'assistance agissent de la même façon².

\footnotetext{
${ }^{1}$ La notion d'accordage que nous utilisons ici doit beaucoup aux travaux fondateurs de Daniel Stern sur les interactions précoces mère-bébé (voir en particulier Stern 1985 ainsi que Gratier 2001). Une définition claire et succincte nous est fournie par Marie-Sophie Bachollet et Daniel Marcelli : « L’accordage affectif est ce moment particulier pendant lequel mère et bébé s'engagent de concert dans un comportement qui traduit le partage d'un état affectif » $(2010,17)$.

${ }^{2}$ Cette hétérogénéité des rôles rituels ressort clairement, par exemple, des descriptions que mobilise Durkheim pour illustrer ses propos sur «l'effervescence collective »: la distinction entre personnes ayant différentes relations de parenté avec le défunt dans les rites de deuil, dont la place singulière qu'y occupe le conjoint survivant (1912, 372-374 ; cf. Moisseeff 2017), ou entre " officiants » et "préparateurs » dans les rites totémiques des Aborigènes australiens (1912, 212-213).
} 
Si une telle complémentarité interactive au service d'une entreprise commune se retrouve bien aussi dans la vie de tous les jours, sa ritualisation la dote de qualités distinctives. Tout d'abord, il est de règle que les pratiques rituelles comportent non seulement une implication corporelle appuyée, mais aussi une sollicitation intense à la fois des sens et des expressions affectives. De ce point de vue, les interactions qui participent de ces pratiques faire ses adieux au défunt, prononcer des vœux ou porter un toast aux mariés, etc. - sont particulièrement marquantes sur le plan des ressentis qu'elles mobilisent. Mais le comportement rituel se singularise surtout par le fait qu'il est à la fois hautement schématisé et irréductiblement complexe. D’un côté, il obéit, en regard des activités de la vie quotidienne, à des contraintes supplémentaires qu'imposent des prescriptions spécifiques. Il faut, en effet, agir de telle ou telle façon, selon tel ordre, etc., pour que l'activité menée en commun - les funérailles, le mariage - soit reconnue comme ayant effectivement lieu comme il se doit. Il en résulte que la complémentarité synergique des actions des uns et des autres s'accomplit de façon spécialement flagrante et soutenue. Les rituels mettent en forme et en acte des petits mondes synthétiques, éminemment cohérents, soutenus par une coordination particulièrement systématique des conduites des participants (cf. Turner 1969). Mais, d'un autre côté, les pratiques rituelles sont caractérisées par une complexité spécifique dont la justification échappe, en partie, aux principes d'intelligibilité ordinaires. Les directives paradoxales et les intentionnalités quelque peu mystérieuses qu'elles mettent en œuvre - s'adresser à un mort, revêtir des habits destinés à n'être portés qu'une fois, manifester une grande intimité avec quelqu'un qu'on connaît à peine, etc. - les rend difficiles à comprendre en termes d'interactions quotidiennes. Ces actes acquièrent cependant, pour ceux qui y prennent part, une valeur et une efficacité propres, en raison des expériences spéciales qu'ils fournissent et des retombées pragmatiques qui leur sont reconnues. Ainsi, si la nature des pratiques rituelles demeure difficile à expliciter, les acteurs leur présupposent néanmoins un sens qui ne leur devient pleinement accessible que lorsqu'ils y participent. Elles s'imposent à eux comme des actions «stipulées » ou " archétypiques » dont ils seraient à la fois les auteurs et pas les auteurs (Humphrey et Laidlaw 1994). En somme, les conduites rituelles se démarquent des façons de faire de tous les jours parce qu'elles sont fixées par la coutume et néanmoins foncièrement équivoques.

Dans de telles conditions propres au rituel, les émotions que donnent à voir les participants sont indissociables des agissements prescrits qu'ils s'efforcent d'adopter. L'expression émotionnelle se réalise en effet dans le cadre de prescriptions ne portant ni directement sur l'état affectif que devraient ressentir les participants, ni sur une représentation purement théâtralisée des sentiments, mais sur des actes conventionnels impliquant l'expression tangible de certains ressentis. Alors que dans l'interaction quotidienne, l'idée qu'on se fait de l'état d'esprit des uns et des autres est le point de départ à partir duquel se déploie un comportement social négocié, dans le cas du rituel ce sont, au contraire, des schèmes de comportement bien définis qui constituent les bases pour l'expression, partiellement idiosyncrasique, des sentiments de chacun. Par exemple, se conformer aux gestes requis consistant à marcher sans jamais croiser les bras et sans regarder en arrière tout en affichant une expression de tristesse n'empêche pas de laisser libre cours à son propre chagrin. Et, réciproquement, l'exécution concertée de ces gestes, sans pouvoir déterminer une convergence des ressentis personnels de chacun, peut contribuer à la susciter. Dans les rites, un rapport circulaire s'établit donc entre comportements prescrits et ressentis individuels, de sorte que, comme l'affirme Marcel Mauss à propos de l' ' expression obligatoire des sentiments » au cours des cérémonies funéraires, «tout [...] est à la fois social, obligatoire, et cependant violent et naturel » $(1921,413)$.

Ainsi, les actes rituels, bien que prescrits, ne sont pas accomplis de façon mécanique. Ils sont informés par une expressivité affective que chacun investit de façon personnelle. Corrélativement, l'articulation coordonnée des comportements différents que poursuivent 
différentes catégories de participants, bien que prévisible, n'a rien d’automatique ; elle est soustendue par l'implication émotionnelle personnalisée de chacun. Nous qualifions de « mutualisation » des émotions cette mise à disposition des vécus affectifs de chacun afin que tous contribuent ensemble au bon déroulement du rituel. Toutefois, c'est en raison même de l'investissement individué qui l'anime que cette mutualisation requiert un travail d'orchestration. Elle exige, en effet, que les participants soient engagés dans un processus continu d'ajustement non pas tant du contenu de leurs expressions émotionnelles respectives (lequel est en principe déterminé par les comportements prescrits qu'ils poursuivent), que de l'intensité de ces expressions. C'est selon nous cette modulation réciproque de l'amplitude de leurs manifestations émotionnelles tout au long de l'exécution des actions rituelles qui permet aux acteurs de s'accorder les uns aux autres conformément aux rythmes affectifs qu'occasionne leur performance conjointe. Or, et c'est là une hypothèse supplémentaire sur laquelle nous reviendrons, ce travail d'orchestration émotionnelle dépend moins des perceptions qu'ont les participants des conduites des uns et des autres, que des sensations corporelles d'ordre cénesthésique que suscitent chez eux ces conduites.

Afin d'étayer les propositions que nous venons d'esquisser, et ce faisant de préciser les ressorts de l'expression émotionnelle particulièrement intense qui se donne à voir au cours de certains rites collectifs, nous allons tout d'abord poursuivre l'exploration ouverte par Durkheim et Mauss concernant la gestion du deuil en considérant les pratiques funéraires actuelles d'une communauté aborigène du Sud de l'Australie. La disparité des rôles tenus par les participants ainsi que la complémentarité de leurs actes sont on ne peut plus claires et persistent de bout en bout. Ce cas nous permettra donc d'illustrer concrètement la forme paradigmatique d'un agencement coordonné de comportements différents qui sous-tend la structuration d'une pratique rituelle collective et, par là, l'expression des émotions à laquelle elle donne lieu.

On se penchera ensuite sur le rite gisaro des Kaluli de Papouasie-Nouvelle-Guinée. Au cours de cette performance, des danseurs-chanteurs venus d'une localité voisine se mettent en scène de manière à évoquer les proches défunts des membres de la localité qui les reçoit, provoquant chez ces derniers des explosions émotionnelles d'une grande violence. Ici aussi, l'accent est mis sur le maintien de l'hétérogénéité des positions qu'occupent les participants visà-vis des défunts. Comme dans le cas des funérailles aborigènes, la coloration affective caractéristique du rituel émerge d'une articulation complémentaire des différents rôles qu'assument les protagonistes, et repose sur un processus d'ajustement réciproque des intensités de leurs expressions émotionnelles respectives.

On considérera en troisième lieu un autre type de pratique rituelle d'une nature sensiblement différente se rapportant aux danses de « découverte de soi » issues du mouvement de développement personnel ayant vu le jour au cours du XXe siècle en Occident. Les cérémonies aborigènes et kaluli sont des actes rituels de facture canonique dont les principes de fonctionnement sont bien connus des ethnologues. Les participants s'y engagent dans des actions prescrites auxquelles ils confèrent une valeur et une efficacité propres. En revanche, les danses de découverte de soi s'apparentent à des ritualités contemporaines dont le fonctionnement reste encore peu travaillé ( $c f$. Houseman 2016). Les participants s'y livrent à des actions qu'ils conçoivent avant tout comme des moyens d'induire et/ou d'exprimer des dispositions qui leur sont enjointes et qui sont supposées avoir une valeur et une efficacité intrinsèques : « spontanéité », « créativité », « ouverture à l'autre », etc. Dans le premier cas de figure, les acteurs s'efforcent de reproduire des comportements que d'autres auraient effectués avant eux (ancêtres et autres représentants de la «tradition »). Dans le second, ils cherchent à expérimenter, par des comportements qu'ils créent ou renouvellent, des façons de penser et de ressentir que d'autres avant eux auraient éprouvées (peuples non occidentaux ou préchrétiens, enfants et autres figures d'une " authenticité » perdue). Toutefois, les dynamiques régissant l'expression émotionnelle sont similaires dans l'un et l'autre cas. Ainsi, dans les danses de 
découverte de soi, où chaque participant s'efforce de « se retrouver » en évoluant librement en compagnie des autres, l'hétérogénéité qui préside à leur participation conjointe se rapporte à la singularité personnelle de chacun. L’agencement complémentaire de leurs agissements respectifs et des expressions émotionnelles que leurs comportements donnent à voir est médiatisé par des interactions affectivement chargées qui impliquent un accordage continu des amplitudes de ces expressions.

Enfin, la présentation succincte d'un rite inaugurant une expédition de vengeance aborigène observée à la fin du XIXe siècle en Australie chez les Aranda permettra de montrer que dans les rituels ayant, par contraste avec les précédents, pour visée explicite de susciter une même émotion chez tous les participants, celle-ci procède, non d'un processus spontanée de contagion affective, mais de leur engagement dans une succession complexe d'interactions prédéterminées qui sous-tend la constitution progressive d'une identification entre eux.

\section{L’expression émotionnelle dans la gestion collective du deuil}

Nous commencerons par considérer l'orchestration des émotions dans les rites funéraires ayant cours de nos jours dans une communauté aborigène du Sud de l'Australie (Moisseeff 2016a ; 2020) ${ }^{3}$. À l'annonce de la mort, les proches du défunt avec qui il a partagé une forte intimité - conjoint, enfants, père et mère, frères et sœurs -, que l’on désignera ici comme les deuilleurs, se mettent à hurler et s'infligent parfois de profondes blessures corporelles. Dans les semaines qui suivent, leur douleur prend la forme d'une prostration plus ou moins marquée qui, la plupart du temps, persiste au-delà de l'enterrement. Cet abattement initial est entrecoupé d'épisodes extrêmement démonstratifs de chagrin et, aussi parfois, de colère, notamment lorsque le décès est inattendu ou causé par des circonstances particulièrement violentes ou traumatiques tels que suicide, meurtre ou accident, nécessitant alors souvent l'intervention des autres.

De nos jours, en raison de la législation australienne, le cadavre est confié aux pompes funèbres qui président à sa mise en bière et à l'inhumation. Le personnel tend à respecter les modalités coutumières aborigènes, en acceptant que plusieurs membres de la famille restent de longs moments et à de nombreuses reprises auprès du défunt, et qu'ils procèdent à certains soins (le coiffer, le maquiller, parfois couper des touffes de cheveux pour les conserver). Ils acceptent aussi de garder le cadavre beaucoup plus longtemps qu'il n'est de règle pour le reste de la population australienne. L'ethos aborigène exige, en effet, que tout soit mis en œuvre pour s'assurer que l'ensemble des personnes susceptibles d'être affectées par la disparition - souvent plusieurs centaines, voire plus, provenant de communautés très éloignées les unes des autres soient prévenues et puissent avoir le temps de s'organiser afin de venir soutenir les deuilleurs et d'assister aux funérailles. Les Aborigènes attendent, en effet, de leurs proches qu'ils soient solidaires avec eux dans le chagrin. Réciproquement, les proches souhaitent ardemment faire montre concrètement de leur solidarité au travers d'une proximité physique et émotionnelle, en acceptant d'être affectés aussi profondément que possible et de l'exhiber ${ }^{4}$.

C’est pour la même raison que la période qui précède l'enterrement est marquée par la transformation des modes ordinaires de sociabilité. Les deuilleurs tendent à se regrouper et à résider ensemble. Certains restent dans leur maison tandis que les plus traditionnels s’installent dans un campement à l'extérieur appelé sorry business camp. Ils sont dans tous les cas rejoints par les membres de leur famille élargie. D’autres visiteurs viennent marquer leur respect en

\footnotetext{
${ }^{3}$ Pour une présentation plus fournie d’un éventail de pratiques funéraires actuelles des Aborigènes australiens, voir Glaskin et al. 2008.

${ }^{4}$ Cf. Tonkinson 2008 ; pour une analyse analogue concernant une population amérindienne amazonienne, voir Allard 2013.
} 
passant quelque temps avec les deuilleurs, et leur apportent nourriture et boissons, parfois même leur glissent de l'argent. Des femmes âgées, qui ne sont pas les plus proches parentes, vont jouer le rôle de pleureuses : par intermittence et à l'arrivée de chaque nouveau venu, elles se mettent à pleurer en chœur de façon conventionnelle et extrêmement bruyante pendant quelques minutes. La puissance et le caractère poignant de ces lamentations sont à la mesure des sensations physiques violentes qu'elles provoquent à chaque fois chez les personnes présentes. Leur répétition inopinée qui interrompt soudainement la reprise des échanges ordinaires relance quant à elle l'intensité des ressentis qui auraient sinon tendance à retomber. La mise en place de l'accordage de l'expression émotionnelle qu'établissent entre elles ces vieilles femmes en pleurant en chœur à l'unisson médiatise donc l'ajustement de l'intensité des ressentis émotionnels de l'ensemble des autres participants. L'agrégation du visiteur au sorry business camp s'effectue simultanément au plan des sensations physiques induites par les pleurs qui l'accueillent et des gestes significatifs auxquels il doit dans le même temps se conformer avec chacun des proches du défunt: il leur serre la main puis ils s'étreignent dans un geste de compassion mutuelle. On l'informe ensuite à voix basse des circonstances de la mort, des dispositions prises tandis que, par la suite, les propos échangés redeviennent d'ordre plus courant jusqu'à ce que retentissent, à nouveau, les hurlements des pleureuses. Ce sont des « alliés » du défunt (certains membres de sa belle-famille) ou des consanguins plus éloignés, et non les deuilleurs, qui fournissent et préparent la nourriture pour les repas et offrent des collations aux visiteurs. Ils participent également aux tâches requises pour les funérailles proprement dites telles que la rédaction et la distribution de la notice nécrologique ou la préparation de la collation qui suivra l'inhumation au cimetière. Ce sont aussi des hommes choisis parmi les consanguins et les alliés du défunt, en raison des relations privilégiées qu'ils ont entretenues avec lui, qui porteront le cercueil. Le jour dit, les deuilleurs et les porteurs, qui occupent tout d'abord le devant de la scène, se signalent par la formalité des habits qu'ils portent: robes, jupes ou pantalons noirs et chemises ou chemisiers blancs ou noirs, ces vêtements étant au besoin empruntés à d'autres ou achetés pour l'occasion (souvent par l'intermédiaire d'associations caritatives). Aujourd'hui, il arrive que certains y associent une pièce de vêtement de la couleur qu'affectionnait le défunt et que des rubans de boutonnière de la même couleur soient mis à disposition de l'assistance en même temps que la notice nécrologique. En dehors des personnes venues en leur qualité d'officiels - policiers et autres figures d'autorité - et du personnel des pompes funèbres qui, eux, se font discrets, les autres participants s'habillent comme à l'ordinaire, c'est-à-dire de manière très informelle.

Le cadavre est exposé dans le cercueil ouvert placé à l'intérieur du lieu de culte chrétien choisi, les funérailles aborigènes s'effectuant toujours, au moins en partie, selon des rites chrétiens. Dès l'entrée dans ce lieu de culte, les deuilleurs et les pleureuses se placent devant le cercueil. Les pleureuses entonnent alors leurs lamentations auxquelles se mêlent instantanément les pleurs et les hurlements retentissants des deuilleuses, certaines faisant mine de se précipiter dans le cercueil, au bord de l'évanouissement, tandis que d'autres les retiennent et les prennent dans leur bras. Au bout de quelque temps, l'officiant chrétien fait son apparition et les lamentations font place au sermon qu'il prononce et aux chants chrétiens entonnés par tous à intervalles réguliers. Les messages envoyés par ceux qui n'ont pu venir sont ensuite lus. Puis le cercueil est fermé, événement qui suscite à nouveau les lamentations des pleureuses et l'explosion des pleurs et des cris des deuilleuses.

Les participants s'acheminent ensuite vers le cimetière, à pied ou en voiture, formant un long et lent cortège que l'on ne peut ignorer en raison de l'affluence. Dans le cimetière, ils marchent silencieusement, en communion, jusqu'à l'endroit où le cercueil est déposé à terre. 
Lorsqu'il est descendu dans la fosse, une nouvelle explosion de pleurs et de cris de la part des pleureuses et des deuilleuses retentit. La veuve, ou une sœur, ou une fille, peut faire mine de s'élancer sur le cercueil, voire s'évanouit à demi. Il arrive ensuite souvent que des proches du mort se mettent à chanter et à jouer de la guitare, en entonnant les chansons que le mort affectionnait particulièrement, ou qu'il avait composées. Puis chacun jette un peu de terre sur le cercueil ou une fleur, et serre la main des deuilleurs. Tout le monde repart du cimetière, de manière plus dispersée qu'à l'arrivée, et se retrouve dans un endroit public - hall communautaire, club sportif, pub - où de petits sandwichs, des gâteaux, des boissons ont été disposés sur des tables. L'ambiance n'est alors plus du tout lugubre. Au contraire, et en contraste total avec l'atmosphère précédente, on rit volontiers et bruyamment, entre autres en évoquant certains événements plaisants ou carrément drôles qu'on a vécus avec le défunt. On s'enquiert des nouvelles des uns et des autres. Même la mère qui vient d'enterrer son fils, et qui hurlait encore de douleur quelques minutes auparavant, participe avec entrain aux discussions.

Quelques heures plus tard, les groupes se dispersent mais les deuilleurs et d'autres membres de la famille tendent à rester ensemble, occasion de questionner les circonstances de la mort et le caractère approprié des dispositions prises pour les obsèques. Il arrive alors fréquemment que des disputes éclatent, et des bagarres plus ou moins sérieuses peuvent prendre place, phénomènes favorisés par la consommation abondante d'alcool. De fait, tout décès tend à raviver les conflits familiaux.

La période collective du deuil que nous venons de considérer débute dès l'annonce du décès et s'achève au terme de la succession des événements qui entourent l'inhumation. Dans l'intervalle, les membres de la communauté concernée contribuent, en fonction du plus ou moins grand degré de proximité qu'ils ont avec le mort, à la mise en place d'une expérience partagée qui s'impose comme une représentation collective de la douleur et de la perte. De ce point de vue, ils constituent, selon la formule d'Arnold van Gennep, une "société spéciale » (1909, 211) qui se caractérise par la restructuration de l'organisation spatio-temporelle de la vie quotidienne. Dans le sorry business camp ou la maison qui en tient lieu, les deuilleurs qui y sont confinés occupent la place centrale autour de laquelle gravitent les autres catégories de participants. Déchargés des tâches ordinaires, assumés par des parents plus éloignés du défunt, ils sont incités à se consacrer entièrement à leur chagrin et à le manifester sur un mode ostentatoire. Les pleureuses participent, quant à elles et comme nous l'avons vu, à l'ajustement de l'intensité des ressentis des uns et des autres. Dans cette première partie de la gestion collective du deuil, le confinement des deuilleurs dans un espace dédié à l'expression du chagrin, inséré dans la communauté des vivants, renvoie à l'association étroite établie entre eux et le défunt : le corps prostré des premiers se substitue en quelque sorte au corps inanimé du mort en faisant écho à son absence, à sa relégation à l'extérieur. C'est la mise à l'écart des deuilleurs et du mort, respectivement au centre et à la périphérie de la communauté, qui soustend la constitution d'une société spéciale temporaire. De fait, dans la chambre funéraire où repose le cadavre, l'intimité physique des deuilleurs avec le défunt est transitoirement prolongée. Le jour de l'inhumation, on assiste à la mise en place progressive d'une distance établie entre eux via le passage dans des espaces publics successifs. Dans l'église ou le temple, leur présence conjointe sur le devant de la scène où trône le cercueil les réunit encore mais de façon nettement moins exclusive et privée. Dans le cimetière, la descente du cercueil dans la fosse opère leur séparation physique définitive qui donne lieu à la dernière scène empreinte de pathos. Le cadavre y demeure tandis que les deuilleurs sont conviés à rejoindre les vivants dans le lieu où prend place la réception qui clôt les funérailles.

L'itinéraire suivi par les deuilleurs, depuis l'annonce du décès, les fait passer d'un lieu d'enfermement, le sorry business camp, à un autre, la chambre funéraire, pour finalement les réintroduire dans des espaces publics de plus en plus ouverts où vont converger, en très grand nombre, divers participants dont la majorité ont des liens plus distancés avec le défunt. La 
formalité des gestes et des attitudes que ces participants adoptent comme « naturellement » est tout d'abord en phase avec l'intensité des émotions exprimées par les deuilleurs, et est orchestrée par les divers tiers présents : pleureuses, porteurs, officiants du culte ou des pompes funèbres. Mais en se substituant aux lamentations proférées par les femmes, les chants, tout d'abord religieux puis profanes, entonnés en chœur par l'ensemble de l'assistance redonnent à celle-ci et, par là, à la communauté des vivants, une place dont elle s'était volontairement abstraite pour marquer la solennité du moment. L'ensemble des participants recouvre ainsi progressivement la liberté d'exprimer ouvertement autre chose que la tristesse en donnant à voir toute la gamme des émotions, depuis la joie jusqu'à la colère, à l'instar de ce qui se passe dans la vie quotidienne. Si, au cours de la période qui précède l'inhumation, c'est à n'en pas douter le chagrin exprimé par les deuilleurs et les pleureuses, qui donne le ton à la mise en place de l'expérience partagée, dès que le mort prend place dans le cimetière, le mouvement s’inverse. C'est au tour des deuilleurs d'ajuster l'expression de leurs ressentis sur celle des autres participants parmi lesquels se fondent les pleureuses, le rôle de celles-ci étant désormais devenu caduque.

Nous voyons que l'expression collective du deuil repose bien sur la complémentarité des comportements que chacun effectue en fonction des relations qu'il entretenait avec le défunt. La prise en charge de ces comportements par les différentes personnes à la fois les affecte intimement, en modelant leur expérience émotionnelle, et modèle leur façon d'exprimer, face aux autres, cette expérience intime. La mise en scène conventionnelle du deuil substitue aux obligations réciproques complexes du quotidien entre le défunt et ses proches auxquelles sa transformation en cadavre a mis fin, les obligations mutuelles définissant les relations prescrites entre les deuilleurs et les autres catégories de participants. Cette réciprocité reconfigurée va se déployer dans des espaces particuliers proprement rituels au sein desquels la temporalité ordinaire est suspendue : tout un chacun doit disposer de suffisamment de temps pour se consacrer pleinement au travail (business) requis pour tisser avec les autres l'expérience du partage collectif des émotions ( $c f$. la prolongation du temps de garde habituel du cadavre imposée par les Aborigènes aux pompes funèbres). Elle est médiatisée par des tiers professionnels ou quasi professionnels, ici respectivement les officiants des pompes funèbres ou du culte chrétien et les pleureuses et les porteurs. Les relations rituellement instaurées, et qui sont cependant en continuité avec celles de la vie ordinaire, se réalisent au travers d'interactions simplifiées par leur formalité dont le moteur est alimenté par les émotions générées par l'exhibition ostentatoire du chagrin. Mais ces émotions ne vont pas dans le même sens pour les deuilleurs et pour les autres. Les intimes du défunt qui sont le plus affectés par sa mort sont incités à extérioriser les émotions internes et intenses qui les habitent. En revanche, ceux qui sont plus éloignés du mort, en étant transformés en spectateurs obligés de cette mise en scène de la douleur associée à la perte, sont mis en contact intime, interne et intense avec leurs propres morts, leurs propres sentiments de tristesse et de perte. La performance rituelle, par le biais de la complémentarité des gestes effectués par ceux occupant des places distinctes vis-à-vis du mort, médiatise par les ajustements réciproques qu'elle implique l'harmonisation de l'intensité des sentiments ressentis par les uns et les autres.

\section{Une remémoration collective et ritualisée des sentiments de deuil}

Chez les Kaluli de Papouasie-Nouvelle-Guinée, les hommes des différentes localités s’invitent à tour de rôle pour exécuter ensemble un rite appelé gisaro qui donne lieu à une autre 
forme de partage d'expérience émotionnelle ${ }^{5}$. La cérémonie se déroule durant toute une nuit à l'intérieur d'une maison communautaire dont l'allée centrale est éclairée par des torches. Les hôtes invitants sont assis, dans l'ombre, de chaque côté de cet espace central sur les plateformes de couchage réservées à chaque famille. Parmi les invités, des danseurs magnifiquement parés, le visage baissé et l'expression fermée, dansent avec une lenteur étudiée d'une extrémité à l'autre de l'allée centrale, tout en chantant à voix basse, accompagnés par des chœurs installés à chaque extrémité. Ils sont habillés en oiseaux, animaux étroitement liés aux esprits des morts. Leurs chants obsédants évoquent, au travers d'images poétiques suggérant l'abandon, la perte et la solitude, le paysage environnant et mentionnent des noms de lieux dispersés sur le territoire de leurs hôtes. Ces derniers, profondément émus, sont ainsi amenés à se remémorer les personnes récemment disparues qu'ils associent à ces lieux. La scène suivante est typique de celles qui ne vont cesser de se répéter durant la nuit (Schieffelin 1976, 23). Un ancien, mis en fureur par le chagrin qui l'avait soudain submergé, se mit à proférer de bruyants hurlements de souffrance et se leva d'un bond. Il se saisit alors d'une torche et en enfonça l'extrémité enflammée dans l'épaule nue du danseur-chanteur qui resta impassible. Puis il se précipita dehors pour gémir sur la véranda. Au petit matin, les visiteurs mirent abruptement fin à la cérémonie :

« Les danseurs, dont les épaules étaient assez sérieusement brûlées, payèrent alors une compensation [servant à faire des parures corporelles] à ceux qu'ils avaient fait pleurer, et tous les visiteurs quittèrent ensemble la maison pour rentrer chez eux. Parce que beaucoup de gens avaient pleuré, la cérémonie fut tenue pour réussie. Certains des visiteurs partirent en gémissant par sympathie pour ceux de leurs parents, parmi les hôtes, qui avaient été envahis par le chagrin » (ibid., 24).

Ici encore, la convention rituelle d'expression des émotions de douleur et de deuil permet aux deuilleurs de faire passer leurs sentiments internes qui les lient au défunt, à l'extérieur d'eux, sur d'autres qui ont charge d'évoquer l'absence du défunt, et notamment la non présence définitive de son corps. Au travers des images lyriques véhiculées par les chants évoquant la solitude et la désolation du paysage, l'absence du défunt, qui a pour pendant l'exacerbation de sa présence dans les souvenirs des deuilleurs, reçoit une expression symbolique à la fois tangible et suffisamment abstraite pour que chacun se les approprie en les associant aux lieux spécifiques où il avait pour habitude de se rendre avec un parent proche aujourd'hui disparu. La présence de l'étranger évoquant ces lieux redouble, en l'actualisant dans l'ici et maintenant publique du rite, le sentiment, pour le deuilleur, de la perte définitive de l'intimité physique et émotionnelle qu'il a autrefois partagée avec le défunt. Mais, en même temps, le comportement rituel, c'est-à-dire conventionnellement prescrit, de pleurer de manière ostentatoire et de brûler le chanteur, sert d'exutoire au chagrin intime.

Les émotions des deuilleurs sont réifiées par les blessures infligées aux danseurschanteurs et échangées contre des présents concrets dont les hôtes vont se revêtir. Les parures corporelles, tout comme les blessures visibles infligées aux chanteurs, extériorisent les blessures internes et invisibles que les chants ont réouvertes. L'émotion personnelle est ainsi objectivée et, en tant que telle, transformée en objet de partage au plan collectif. Ce partage de la tristesse participe, en effet, au cycle d'échanges qui lie les différents groupes kaluli les uns aux autres, tout comme un tel partage participe, chez les Aborigènes, de l'ensemble des obligations réciproques que se doivent les différents membres participant d'un même réseau de parenté élargi qui résident dans diverses localités réparties sur le territoire australien. De fait, les ressorts de l'expression collective des émotions dans le rituel kaluli renvoient, comme dans le cas aborigène évoqué précédemment, à la volonté expressément acceptée - les chanteurs ont

\footnotetext{
${ }^{5}$ Pour l'ethnographie originale et une analyse plus complète de ce rite, voir Schieffelin 1976 ; Feld 1982 ; Munn 1995.
} 
répondu positivement à l'invitation formelle que leurs hôtes leur ont adressée afin qu'ils exécutent ensemble un gisaro, et ces derniers répondront, à leur tour ultérieurement, à une telle invitation - de manifester publiquement, de manière ouverte et ostentatoire, leurs douleurs intimes et d'être profondément affectés par les émotions des autres.

Notons que le rôle tenu par les pleureuses dans le cas aborigène revient, dans le gisaro, aux danseurs-chanteurs : ils jouent le rôle de tiers médiateurs de l'ajustement des émotions entre les individus immédiatement affectés par les émotions liées à la perte d'un proche (les deuilleurs) et les spectateurs affectés de façon consécutive en raison de l'exhibition du chagrin des premiers. L'expression fermée des danseurs-chanteurs au cours de leur performance, allant de pair avec la lenteur de leurs mouvements et la tonalité basse de leurs chants tristes et lancinants, participe de la tension ascensionnelle ressentie par l'ensemble des participants. Cet effacement conventionnel de l'expression de leurs émotions atteint son acmé lorsqu'ils font montre d'une impassibilité ostentatoire au moment où ils sont brûlés. Il dénote aussi la nécessité protocolaire, au cours du rite, de ne pas usurper la place centrale conférée aux deuilleurs qui sont alors seuls incités à faire montre, à l'inverse de manière explosive, du ressenti vécu dans leur for intérieur, attestant ainsi de la réussite du rite. Ce qui ne veut pas dire que les exécutants invités, au même titre que les autres spectateurs, ne ressentent rien mais qu'ils n'ont pas alors à l'exhiber. Par contre, une fois le rite achevé, ils sont libres d'exprimer leur propre ressenti de chagrin comme l'illustre le fait que certains visiteurs partent en gémissant "par sympathie » avec ceux qu'ils ont fait souffrir. La clôture du rite marqué par le don des présents faits par les visiteurs à leurs hôtes leur permet de recouvrer leur capacité à donner libre cours à des émotions sur un mode plus ordinaire, c'est-à-dire celui de la vie quotidienne, non contraint par les exigences de l'organisation des interactions proprement rituelles. L’impassibilité performée participe de la structure complémentaire des positions occupées par chacun au cours du rite : il y a bien ajustement mais non confusion des ressentis à partir d'une distinction des places clairement établies. Et cette complémentarité des positions des uns et des autres s'inversera lors des gisaro ultérieurs exécutés dans la localité des visiteurs.

Chez les Aborigènes, comme dans nombre d'autres sociétés, lors de la période collective du deuil qui précède la séparation définitive des deuilleurs d’avec le corps de leur défunt, les salves de chagrin ostentatoire que les conventions culturelles sollicitent de leur part interrompent leur prostration initiale, associée à leur sidération ou anesthésie affective dont, ici comme ailleurs, ils sont l'objet. Si les autres membres de la communauté "sortent", immédiatement après les obsèques, de la société spéciale, située entre les vivants et les morts, consacrée au partage collectif de la tristesse, la période de deuil se prolonge généralement bien au-delà pour les proches. Leur prostration est alors encouragée et les situe du côté du cadavre de leur défunt jusqu'à ce qu'ils soient réagrégés à la société des vivants par l'exécution d'un

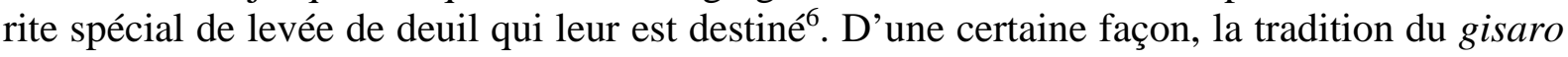
où ce sont les danseurs-chanteurs en tant que tiers non intimes - ils résident habituellement dans une autre localité - qui assument l'impassibilité marque on ne peut mieux la réintégration des deuilleurs dans la société des vivants : elle notifie de façon spectaculaire leur transformation en sujet à même d'imposer activement aux autres leur chagrin et de le convertir en valeur d'échange participant des obligations réciproques entre vivants ; et c'est bien en tant que tels que les deuilleurs sont rétribués.

Le gisaro est, de fait, consacré au partage collectif d'une expérience émotionnelle prenant acte de ce que la mort fait aux vivants. Il le fait au travers d'une organisation conventionnellement préétablie qui comporte une césure introduite dans le cours de la vie sociale ordinaire, généralement diurne, au travers de la mise en place d'une temporalité suspendue - toute une nuit - et d'un espace spécialement apprêté pour l'occasion. Celui-ci est

\footnotetext{
${ }^{6}$ Pour la description d'un tel rite chez les Aborigènes, cf. Moisseeff 2017.
} 
composé d'un centre, seul illuminé et délimité par les deux chœurs, réservé à la performance rituelle proprement dite, et d'une périphérie où règne l'obscurité composée d'espaces privés où sont assis les hôtes. La performance est ponctuée par les appariements successifs d'un danseurchanteur avec le deuilleur affecté par son exécution, celui-ci passant de l'ombre à la lumière, de l'intimité familiale au public, à l'instar des affects du deuil : réparties respectivement entre un visiteur-performeur et un hôte, retenue et décharge émotionnelles extrêmes se renforcent l'une l'autre. Nous posons que ce sont ces appariements qui jouent le rôle d'opérateur du partage émotionnel, entre l'ensemble des personnes présentes, des divers ressentis négatifs, en creux et en plein (anesthésie affective, tristesse, colère) que les défunts induisent chez leurs proches.

C'est, en effet, au travers de ce type d'interactions dyadiques à la complémentarité particulièrement bien accordée que ces ressentis, dont les deuilleurs font l'expérience dans leur for intérieur, sont mis en circulation au sein du collectif disparate rassemblé pour un gisaro. Ils sont réifiés au moyen de procédures artéfactuelles qui permettent, d'un côté, de les distinguer les uns des autres et, de l'autre, de les conjoindre au sein des couples chanteur-deuilleur, c'està-dire de les distribuer entre des acteurs occupant des positions distinctives vis-à-vis des absents présentifiés par une évocation esthétique qui anonymise ces derniers et qui, de ce fait, participe à la réappropriation des affects de deuil par l'ensemble des participants. Ainsi, la décharge émotionnelle des personnes les plus affectées, suscitée par les conditions spécifiques mises en place par le rituel - structuration spatio-temporelle, foisonnement symbolique, formalisme interactionnel exceptionnel - induit, chez les autres participants, des sensations physiques cénesthésiques (infra) auxquels l'ethos propre à la culture concernée fait correspondre un style d'attitudes expressives qui, s'il ne préjuge en rien des ressentis réels plus psychologiques des participants, aboutit néanmoins à une mutualisation collective des émotions.

Les rites funéraires « exotiques » que nous venons d'examiner requièrent, de la part des participants, un mode de participation caractéristique qui, aussi étonnant que cela puisse paraître, est comparable à celui qui régit un ensemble d'activités très « tendance » aujourd'hui, en Occident, à savoir, les pratiques collectives de développement personnel prenant appui sur une attention particulière portée au vécu corporel tel que la respiration, le chant, la danse, ou tout autre type de gestuelle spécifique. En effet, l'intensification émotionnelle qu'elles mettent en œuvre pour offrir à tout un chacun la possibilité de se joindre à d'autres afin de «se découvrir » se fonde, là encore, non pas sur une homogénéisation du positionnement des participants, mais sur l'articulation, rituellement médiatisée, de leurs différences où l'appariement en couples au sein du collectif est également mobilisé. C'est ce que nous allons maintenant tenter de montrer en nous intéressant, plus particulièrement, aux groupes de danse centrés sur la découverte de soi.

\section{La gestion collective de la découverte de soi par la danse}

Selon les sites Internet et les prospectus qui leur sont consacrés, les danses dites « libres », « spirituelles » ou « extatiques » (5 Rythmes, Danse Médecine, Biodanza, Open Floor, Chakradanse, Danse Méditative, etc.) invitent « chacun à vivre la rencontre avec soimême, avec l'autre et avec l'environnement, à travers l'émotion et le plaisir du mouvement » afin de favoriser "l'expression de chacun de manière authentique, spontanée et reliée à notre essence profonde », et de « développer notre plein potentiel ».

Ces danses de découverte de soi, comme on les appellera ici ${ }^{7}$, réunissent entre quinze et cinquante individus lors de séances hebdomadaires de deux heures. Les pratiquants, guidés par

\footnotetext{
${ }^{7}$ Les faits rapportés dans cette section ont été recueillis lors d'un travail ethnographique d’octobre 2014 à mai 2016 consistant pour l'essentiel en la participation à une centaine de séances de Biodanza et de 5 Rythmes, principalement en région parisienne. Cette recherche, financée en partie par le projet Reassembling Democracy:
} 
les indications d'une animatrice ou d'un animateur, dansent sans parler en s'inspirant des rythmes et tonalités d'une sélection musicale préparée à l'avance. Chaque séance est généralement constituée d'une entrée en matière douce suivie par une accélération de la musique et des mouvements, un moment d'apogée, puis un ralentissement progressif. Dans certaines de ces pratiques, les participants évoluent librement: ils dansent en solitaire, forment des partenariats transitoires avec d'autres danseurs, et prennent part à des figures impliquant le concours de tous (comme se mettre tous en cercle ou traverser la salle de part en part). Dans d'autres, ils s'engagent dans une série de propositions chorégraphiques : danser ensemble dans une ronde, marcher seul ou à deux en étant "pleinement présent », synchroniser ses mouvements avec ceux d'un partenaire. Dans tous les cas, les danseurs sont incités à bouger chacun à leur façon car ce ne sont pas les mouvements en eux-mêmes qui importent, mais les sentiments et les états d'esprit qu'ils permettent d'exprimer et les expériences qu'ils peuvent susciter. Comme l'explique Gabrielle Roth, la créatrice des 5 Rythmes, dans une de ses vidéos promotionnelles : "It's not about how you look, it's about how you feel. [...] Whatever you're feeling, surrender to it. Move with it. Breathe into it. And it will change ».

Conformément aux annonces promotionnelles, ces pratiques invitent ceux qui y prennent part à éprouver et à donner à voir, par leur danse, certaines manières de ressentir et d'agir tenues pour exemplaires : la spontanéité, la créativité, l'authenticité, l'ouverture à l'autre, etc. Diverses injonctions tacites ou explicites aident les participants à «retrouver» ces dispositions régulièrement convoquées mais jamais clairement définies : l'aménagement d'un espace-temps protégé, rendu chaleureux et accueillant par des lumières tamisées et des étoffes de couleur, des musiques associées à certaines façons de bouger, l'entrain expressif du mouvement dans une situation où il est interdit de se parler, et en symétrie inverse, le discours omniprésent de l'animatrice. Ce dernier prend la forme de brèves instructions énigmatiques ( « invitez-vous à bouger... ", " suivez vos pieds... ", " ressentez l'énergie qui monte dans votre corps », « voilà... relâchez... oui... oui c’est ça... »), alternant avec des flots de paroles émaillés d’images puissantes («laissez exprimer le feu qui est en vous », « c'est comme marcher sur des nuages ») et de slogans accrocheurs (« ne rien forcer, ne rien retenir »). Les danseurs accueillent ces directives bien moins en tant qu'énoncés dont il conviendrait de comprendre le sens exact (d'ailleurs, elles ne sont souvent que partiellement audibles), que comme des autosuggestions véhiculées par la voix d'un autre. En effet, les paroles de l'animatrice visent avant tout à accompagner les danseurs en suscitant chez eux une attention accrue à leurs ressentis propres, afin de les aider à repérer des indices de continuité entre, d'un côté, leurs réflexions et sentiments et, de l'autre, leurs mouvements et sensations corporelles. Les participants, ainsi soutenus et guidés, cessent de vivre leur enveloppe charnelle comme un fardeau contraignant et sont alors à même de s'aventurer à mobiliser leurs corps afin d'expérimenter d'autres manières d'être.

Mais le cheminement qu'expérimentent les participants est loin d'être simple. Le caractère paradoxal des consignes qui leur sont adressées - être volontairement spontané, s'efforcer d'agir authentiquement, etc. - les conduit à percevoir avec une vigilance accrue les écarts existants entre leurs pensées, ressentis et mouvements effectifs et ceux auxquels ils se doivent d'aspirer. Les injonctions auxquelles sont soumis les danseurs les amènent ainsi, de manière répétée, à vivre des moments de désorientation et de malaise. Cherchant à « déconnecter mon mental » afin de puiser au mieux dans mon potentiel créatif, je suis consterné de constater que je répète inlassablement les mêmes gestes. Déterminé à retrouver une façon de bouger qui me serait naturelle, je suis gêné de me voir copier subrepticement les mouvements

Ritual as Cultural Resource (REDO) de l’Université d’Oslo, a également bénéficié des observations issues du terrain doctoral de Marie Mazella di Bosco (Université Paris Nanterre). Certaines des données et des idées utilisées ici figurent sous une forme différente dans d'autres publications (Houseman, Mazzella di Bosco et Thibault 2016, Houseman et Mazzella di Bosco 2020, Houseman 2020). 
d'un autre. Voyant que mon invitation à danser reste sans réponse, je m’interroge : mon geste de sollicitation serait-il passé inaperçu ? A-t-il été jugé trop abrupt, ou déplacé ? En même temps, l'impossibilité de rester simple spectateur, la musique qui ne s'arrête jamais, les propos encourageants de l'animatrice et les agissements incessants d'autres danseurs incitent les participants à dépasser ces achoppements en se hasardant à effectuer d'autres gestes, en changeant de rythme, en se déplaçant dans la salle, en adoptant une attitude différente, etc. Puis arrive un moment, occasionné par un coup de fatigue, un deuxième souffle inattendu, un changement de musique, un regard complice ou un mouvement parfaitement coordonné à celui d'un voisin, où cette réflexivité exacerbée se distend. La sensation physique de relâchement qui en résulte apporte à la fois un plaisir intense et, dans un second temps, la réalisation qu'on est en train de se vivre et de vivre ses interactions avec d'autres différemment.

Lors de ces moments de grâce fugaces qui, de fait, surgissent fortuitement (c'est-à-dire spontanément, naturellement, authentiquement), c'est comme si l'aptitude ordinairement voilée des danseurs à " se sentir plus intensément vivant » (comme disent les adeptes de la Biodanza) éclatait au grand jour. Les participants ont alors l'impression de vivre quelque chose d'exceptionnel, de découvrir un aspect jusqu'alors masqué de ce qu'ils seraient à même de devenir. Mais, en général, au bout de quelques minutes, l'élation s'éteint, en raison de la fatigue, de ratées chorégraphiques, d'une familiarité croissante avec son partenaire, de sentiments parasites de rivalité ou d'attirance sexuelle, etc. On reprend alors l'exploration sensible et conventionnelle de soi par la danse, mais lestée d'expériences inédites d'un " soi véritable » dont les images et les discours, emphatiques et quasi-liturgiques, des flyers et des sites Internet ( " trouver et vivre le plus profond de ce que vous êtes », etc.), tout comme les propos des animatrices, sont des représentations collectives particulièrement saillantes.

Dans les rites funéraires, l'hétérogénéité qui préside à la participation conjointe des deuilleurs renvoie avant tout à leurs différences sociales préalables : certains agissent en tant que membres de la proche famille du mort, d'autres comme des parents plus éloignés, ou comme des alliés, ou des voisins, etc. En revanche, dans les danses de découverte de soi, l'hétérogénéité se rapporte avant tout à la singularité personnelle de chacun. Pendant la séance, les participants s'efforcent de se défaire de leurs rôles sociaux ordinaires (ceux de parent, d'ami, de voisin, de collègue, etc.) pour agir et interagir en tant que simples individus indépendants. C'est en tant que tels que chacun s'attache à « retrouver » et à faire l'expérience, par la danse, de valeurs essentielles - la spontanéité, la créativité, l'authenticité, etc. - qu'il aurait « perdues » au cours d'une socialisation normative et uniformisante. En personnifiant d'une manière distinctive ces valeurs archétypiques, chacun peut éprouver à la fois corporellement et « en pleine conscience », son « potentiel », son aptitude à « devenir soi-même ». De fait, au fur et à mesure que les danseurs se risquent à expérimenter ces personnifications, en les façonnant avec leurs manières d'être et leur sensibilité propres, en les explorant avec divers partenaires dans différentes circonstances, un renversement s'opère. Progressivement, les pratiquants s'imposent, vis-à-vis d'eux-mêmes et des autres, moins comme des personnes qui s'attachent à incarner certaines qualités, que comme des individus aptes à manifester ces qualités en tant qu'aspects jusqu'alors méconnus d'eux-mêmes. Donner à voir son inventivité, sa capacité à s'émouvoir intensément, son ouverture à l'autre, etc., devient ainsi la démonstration que ces dispositions exemplaires peuvent être « retrouvées ».

En même temps, ces pratiques sont foncièrement collectives ${ }^{8}$. Si chacun y est avant tout attentif à son propre épanouissement, c'est en compagnie d'autres individus qui font de même,

\footnotetext{
${ }^{8}$ D'un point de vue sociologique, la population des danseurs correspond à un profil large mais assez bien défini rappelant celui de la « petite bourgeoisie nouvelle » identifiée par Pierre Bourdieu (1979, 429-431 ; voir aussi Altglas 2014). Ce sont, en très grande majorité, des Blancs appartenant aux classes moyennes urbaines, qui ont en moyenne 40 ans (très peu ont moins de 25 ans) et dont les trois quarts sont des femmes. La moitié exercent des
} 
dans une ambiance marquée par le respect mutuel et la connivence affectueuse. Il est de rigueur, par exemple, de remercier chaleureusement (mais silencieusement) ses partenaires de danse, et de se montrer attentif à ceux qui vous entourent. Aussi, les expériences intenses des uns et des autres, notamment dans le cadre de partenariats passagers, s'accompagnent souvent d'un puissant sentiment de partage qui peut se traduire par de longues étreintes. De ce point de vue, l'importance des cercles de paroles sont également à souligner. A la fin de chaque séance (au début dans la Biodanza), les participants sont conviés à se réunir en cercle en se tenant les mains. L'animatrice parlera alors brièvement des bienfaits de la danse et invitera les participants à respirer profondément tous ensemble (" on inspire la gratitude, on expire la gratitude »), à « chercher le regard des autres danseurs », et à " partager quelque chose avec le groupe ». La séance est ainsi clôturée par l'injonction explicite d'une prise de conscience collective au cours de laquelle « le groupe » fait souvent l’objet de remerciements émus.

Soulignons toutefois que si bon nombre des danseurs ne sont pas des inconnus les uns vis-à-vis des autres, il ne s’agit pas non plus de proches (amis ou apparentés). Si les pratiquants les plus assidus connaissent certains des éléments, superficiels, de la vie des uns et des autres (prénom, lieu de vie, profession, etc.), tous ou presque resteront de simples connaissances. En somme, et à de très rares exceptions près, ceux qui vivent des moments physiquement et émotionnellement intenses en dansant ensemble restent ce que Karen Fingerman (2009) appelle des « inconnus conséquents » (consequential strangers), c'est-à-dire des individus dont on peut beaucoup apprécier les qualités dans le contexte de certaines interactions bien circonscrites, mais avec lesquels on se sent libre de ne pas entretenir de relations plus familières ${ }^{9}$. C'est que l'objet de ces pratiques n'est pas d'encourager les participants à devenir des intimes, mais de permettre à chacun, par l'intermédiaire des autres dont il est important précisément qu'ils ne soient pas des intimes, d'éprouver de manière concrète et sensible son aptitude à devenir intimes avec les membres de son entourage personnel. On attend ainsi des danseurs qu'ils puissent « désencastrer » (Giddens 1991, 18) les expériences exceptionnelles qu’ils y vivent du « contexte local » de la séance afin d'en tirer profit dans leur vie de tous les jours.

Les paroles de l'animatrice et les attitudes des pratiquants plus expérimentés sont des repères normatifs importants pour la sociabilité particulière - à la fois intime et distanciée - que mettent en œuvre ces danses. Toutefois, cette sociabilité se donne surtout à voir dans les interactions entre danseurs, notamment au cours des multiples partenariats passagers auxquels ils sont implicitement et explicitement conviés. Les danses de découverte de soi, on l'a vu, suscitent chez les participants une conscience de soi appuyée et maintes fois réitérée en toile de fond, ponctuée par des moments exaltants de relâchement. Or, l'appariement impose une interaction plus systémique dans laquelle ces deux types d'expérience - l'attention à soi soutenue et le «lâcher prise » - sont toutes deux renforcées. D’un côté, la vigilance introspective des danseurs est continuellement relancée par les réactions du partenaire, de sorte que leurs efforts sont d'autant plus facilement parasités par des pensées et des ressentis dissonants relevant du registre des relations quotidiennes : une attirance physique, un sentiment d'antipathie, une gêne, une réaction de rivalité, un moment d'ennui, etc. Chaque hésitation, chaque initiative - telle façon de se placer vis-à-vis du partenaire, d'imiter ses mouvements, de chercher son regard, etc. - peut facilement devenir l'indice d'une mésentente performative, et du coup, d'une défaillance interpersonnelle que les danseurs se doivent de surmonter ensemble. De l'autre, les sensations de jouissance physique, d'intégration harmonieuse et de prise de

professions relevant du soin, du conseil ou de l'enseignement ; environ un quart exercent une activité artistique, et les autres ont des profils très variés (étudiants, cadres ou employés d'entreprise ou de la fonction publique, etc.).

${ }^{9}$ Ainsi, les rares participants qui sont en couple dans la vie se montrent attentifs à ne pas exhiber leur intimité quotidienne. Quand certains dérogent à la règle - lorsqu'ils ne cessent de danser ensemble, lorsqu'ils se font des câlins, lorsque l'un repousse de manière appuyée l'invitation de l'autre - l'irruption de leur intimité introduit un sentiment de gêne qui interrompt la fluidité habituelle des interactions. 
conscience d'un « soi véritable ", qui surgissent lors des moments de grâce, sont démultipliées par un effet de réverbération : chacun voit, comprend et apprécie le fait que l'autre voit, comprend et apprécie, et cet effet miroir augmente d'autant plus le sentiment d'un partage fluide et cohérent.

Or, cette intensification affective ne procède pas d'une convergence des dispositions respectives des danseurs ou d'une similarité dans leurs façons d'agir. Elle repose plutôt sur une prise en compte systématique et attentionnée des différences qui les séparent. Mazzella di Bosco (sous presse) restitue et analyse en détail, dessins à l'appui, les enjeux concrets des rencontres dansées, et les processus constants de négociation et d'ajustement présidant aux partenariats éphémères qui se nouent, se développent et se délient :

« C’est donc tout un accordage progressif, fondé sur une grande attention portée sur l'autre, sur ce que l'on en perçoit, mais aussi sur ses propres mouvements et ressentis. Un ajustement opéré par de subtils et fugaces jeux de regards (car tout va très vite) qui se portent visiblement sur certaines parties du corps en mouvement chez les deux partenaires, par des postures et orientations du corps, par des gestes qu'on tente de faire répondre à ceux de l'autre, par des contacts tactiles prolongés ou au contraire interrompus. Dans un état d'hyper-sensibilité et hyper-réactivité, les partenaires adaptent en continu leurs gestes l'un à l'autre et à tous les éléments ambiants (musique, guidage, autres danseurs alentour) ».

Il est important de souligner que cette coordination à la fois comportementale et affective ne se résume pas à une articulation mécanique relevant d'une perception claire que chacun aurait des gestes et manifestations émotives de l'autre. Il s'agit d'une collusion à la fois plus exigeante, plus émouvante et plus aventureuse. Bien que répondant à l'injonction de "retrouver » certaines dispositions jugées exemplaires, elle est en fait animée par l'implication personnelle des deux partenaires, et orientée par les ressentis et réactions irréfléchies que provoquent chez tous les deux les agissements de l'un et l'autre. Tout comme dans le cas des pratiques funéraires décrites plus haut, l'ajustement réciproque de l'intensité de leurs expressions émotionnelles respectives fournit aux partenaires de danse une trame commune leur permettant d'agencer avec d'autant plus de succès leurs actes de manière complémentaire. De ce point de vue, il est significatif que ces danses soient portées par une succession de rythmes et de tonalités musicales différentes: dans le cas du 5 Rythmes, par exemple, il s’agit de " fluide ", "staccato », " chaos », " lyrique » et " quiétude ». Les pratiquants sont ainsi incités à expérimenter leurs aptitudes à accorder l'intensité des manifestations affectives des uns et des autres dans le cadre de registres interactifs successifs très différents.

Soulignons à quel point les normes de créativité, de spontanéité et de respect des particularités de chacun qui sous-tendent ici l'accordage entre partenaires s'opposent à celles régissant la socialité propre aux véritables intimes. Ces derniers tendent à se retrouver dans une répétition consciemment assumée, et de façon corrélative, dans une homogénéisation de leurs vécus corporels et affectifs qui donne à chacun une emprise sur l'autre. C'est que leurs corps et leurs émotions sont constitutifs de la relation qui les unit, tandis que pour les partenaires de danse, leurs corps et leurs ressentis sont avant tout des moyens qu'ils mobilisent de manière réflexive afin d'expérimenter quelque chose de ce qu'une relation pourrait être ailleurs mais qu'elle n'est pas dans l'ici et maintenant de la séance. Préservant ainsi l’hétérogénéité de leurs positions et points de vue respectifs, les danseurs sont libres d'explorer, physiquement et émotionnellement, les dimensions épanouissantes de la proximité interpersonnelle, sans que cela ne porte atteinte, dans ce cadre strictement défini, à leur autonomie individuelle.

On comprend alors l'impératif d'interagir avec une grande variété d'individus : danser toujours avec la même personne est fortement découragé et les participants sont encouragés à se déplacer pour trouver de nouveaux partenaires. Ces multiples partenaires, avec leurs 
manières distinctives de bouger, d'échanger des regards, de toucher, d'exprimer des émotions, etc., offrent à chacun autant de façons d'éprouver sous différents angles le potentiel qui lui est propre pour entrer en relation avec les autres. "L'autre c'est une lumière ", nous a une fois expliqué une animatrice, " comme une lampe torche qui m'éclaire, qui me révèle à moimême ». Et si l'autre est une ressource pour soi-même, chacun l'est aussi pour d'autres, ce qui est aussi source de satisfaction. De fait, les participants expriment aisément le plaisir qu'ils ont pu ressentir de voir des émotions émerger d'une interaction, de voir apparaître un sourire sur le visage d'un partenaire qui était au départ mal à l'aise, etc. Être témoin, savoir que l'autre me prend pour témoin, devient ainsi un moyen pour chacun d'expérimenter ses propres capacités d'ouverture et d'empathie.

De ce point de vue, les partenaires de danse de découverte de soi entretiennent entre eux un rapport qu'il est intéressant de comparer à celui mis en œuvre dans un gisaro. Au sein de chaque couple chanteur-deuilleur kaluli, le visiteur est une ressource pour l'hôte à qui il permet d'externaliser son émotion interne amenant les autres participants érigés en témoins à être consécutivement, et bien que de façon distinctive, affectés. Dans le cadre d'un gisaro ultérieur dans une autre localité, ces rapports complémentaires s'inversent, l'hôte prenant la place du chanteur-ressource et vice versa. Dans les danses de découverte de soi, c'est au cours d'une même séance que les participants sont amenés à être alternativement et par moment simultanément ressource, témoin, et destinataire des émotions suscitées, ici de tonalité joyeuse. En ce cas, les rôles si clairement répartis entre les partenaires des couples dans le gisaro sont endossés simultanément et alternativement par tous les danseurs. L'hétérogénéité comportementale et expressive se rapporte plutôt ici à l'identité personnelle singulière que chacun est censé mettre en évidence dans sa danse et au cours de ses partenariats avec d'autres danseurs.

En explorant par différents biais l'expressivité affective de ses propres mouvements, en s'ajustant aux mouvements d'autres danseurs au cours de partenariats passagers, en mettant à profit les ressources relationnelles qu'offre la présence de ces multiples partenaires et du groupe, en agissant soi-même comme ressource pour d'autres, les participants font l'apprentissage d'un assouplissement à la fois physique et émotionnel. En d'autres termes, ils acquièrent une aptitude à s'éprouver sans cesse à nouveaux frais. Plus précisément, en médiatisant un va-et-vient continu entre une réflexivité exacerbée et un lâcher prise jubilatoire centrés sur les sensations corporelles et les ressentis affectifs, ces pratiques apporteraient à chacun l'expérience d'une prise de possession de son corps et de son vécu émotionnel. Au cours de ces danses, corps et émotions sont expérimentés, moins comme des présences ou des forces extérieures à soi que l'on est destiné à subir, et plus comme des dimensions utiles de sa personne sur lesquelles une certaine emprise est possible. Ainsi, les valeurs exemplaires souvent convoquées en lien avec ces pratiques - spontanéité, authenticité, créativité, ouverture à soi et à l'autre, etc. - renverraient, en fin de compte, à un sentiment d'autonomie et à une plus grande confiance en soi en compagnie d'autres personnes. Dans cette optique, on peut comprendre les danses de découverte de soi comme contribuant, à leur manière, à la consolidation et à la transmission d'une certaine conception occidentale du socius selon laquelle le potentiel relationnel des individus reposerait sur l'accomplissement volontaire de chacun.

Les danses de découverte de soi sont marquées par une sociabilité où intensification émotionnelle et distance interpersonnelle vont de pair. En promouvant l'expression démesurée et hautement réglementée d'une familiarité corporelle et affective, mais en l'absence des attaches contraignantes propres aux interactions entre intimes, ces danses instaurent des conditions relationnelles qui rappellent par bien des aspects l'intimité distanciée ou anonymisée qui préside à la rencontre avec les «psy ». En effet, ces derniers assument le rôle de «tiers médiateurs » en raison de « la qualité de dépositaire de l'intime de chacun que la société leur a déléguée depuis l'assomption de l'idéal individualiste, précisément en vertu du fait qu'ils ne sont 
pas des intimes » (Moisseeff 2012, 63 ; 2016a). Or, au cours de ces danses, chacun est appelé à assumer le rôle de tiers médiateur pour les autres, l'animatrice l'assumant pour tous. Cela ne veut pas dire que ces pratiques sont assimilables à une démarche psychothérapeutique ; leurs animatrices s'en défendent d'ailleurs explicitement. Toutefois, bien qu'émotionnellement et corporellement investies, les relations qui s'y nouent entre participants, à l'exemple de celles qui s’instaurent entre les psychothérapeutes et leurs patients, ont pour visée, non pas l'identification des uns avec les autres, mais au contraire la préservation de l'autonomie de chacun. Ainsi, dans le microcosme que représente l'espace de danse, se réalise, en quelque sorte, la mise en forme et en acte d'un modèle social utopique cher à la sensibilité occidentale contemporaine : celui d'un « individualisme collectif » admettant la coexistence sans heurt des sentiments de solidarité et de plénitude personnelle. Corrélativement, le régime d’interaction que mettent en œuvre ces danses fait naître un vécu émotionnel spécifique, celui d'une intimité simultanément exacerbée et impersonnelle, qui témoigne à la fois de l'intensité de l'expérience que partagent les danseurs et de l’altérité qui les sépare.

Au fur et à mesure qu’avance la séance de danse, les participants se déplacent davantage, passant d'un partenaire à l'autre, échangeant des regards et ajustant leurs mouvements différemment avec chacun. Leurs corps s'expriment de plus en plus librement, et lors d'épisodes de lâcher prise de plus en plus fréquents, certains peuvent aller jusqu'à crier ou éclater en sanglots ; d'autres deviennent joueurs, formant des cercles de danse à plusieurs, ou sautillant autour de la salle. Un sentiment de reconnaissance réciproque et de connivence joyeuse devient palpable, s'exprimant dans la plus grande expressivité des mouvements comme dans les tendres étreintes et les gestes consciencieux de remerciement qui marquent la fin des partenariats. Le sentiment que partagent alors les danseurs n'a rien d'un élan fusionnel. Tout comme l'accordage entre partenaires qui en est le creuset interactif, il représente une mutualisation émotionnelle de type spécifique, régie par des prescriptions que nous qualifierons volontiers de « rituelles » et soutenue par l'engagement volontaire de chacun. Cette situation est similaire à bien des égards à celle que l'on retrouve dans des pratiques rituelles qui cherchent explicitement à susciter l'homogénéisation du positionnement des divers participants afin qu'ils constituent ensemble un collectif de semblables mieux à même d'unir leurs efforts pour affronter, lors d'une compétition ou d'interventions plus offensives (guerres, vendettas), une partie adverse.

C’est un rite canonique de ce type que nous allons maintenant considérer rapidement.

\section{La construction rituelle d'un collectif de semblables}

Le rite en question, dont les données ont été recueillies à la fin du XIXe siècle par Baldwin Spencer et Francis Gillen (1927, 443-53), est celui qui inaugure une expédition de vengeance chez les Aranda, un groupe aborigène australien dont l'ethnographie a fortement influencé, entre autres auteurs, Durkheim et Mauss.

Lorsqu'un homme souhaite venger la mort d'un proche, il se sert de la ceinture qu'il a tressée avec les cheveux qu'il a coupés sur le cadavre du défunt et qui est censée contenir l'essence spirituelle de celui-ci. On attribue à cet objet le pouvoir de dessécher jusqu'à ce que mort s'ensuive le corps de l'ennemi. Elle aurait, en outre, l'aptitude à potentialiser ses effets mortifères en inoculant un même désir ardent de vengeance aux individus avec lesquels elle est mise en contact, en l'occurrence les acolytes que le justicier a choisis pour l'accompagner. C'est à cette fin que l'initiateur de l'expédition procède à un cérémonial composé d'une suite d'interactions spécifiques. Il commence par frotter son pénis subincisé ${ }^{10}$ sur la paume de leur main. Ce geste, habituellement utilisé pour apaiser les conflits (Berndt et Berndt 1945, 261),

\footnotetext{
${ }^{10}$ La subincision consiste à inciser la face inférieure du pénis de la racine des testicules jusqu'au méat urinaire. Cette opération est pratiquée au cours de l'initiation masculine.
} 
instaure aussi une intimité extrêmement forte entre le vengeur et chacun de ses partenaires. Puis c'est la ceinture, c'est-à-dire ce qui le lie lui-même au défunt (père ou frère) avec qui il partage un même sang, à la différence des autres participants, qu'il frotte sur leur estomac. Il instaure ainsi une intimité entre le défunt et l'ensemble de ses acolytes, ce qui va les rendre aptes à supporter sans périr la conjonction qu'il établit ensuite entre eux tous par l'intermédiaire de la ceinture. Il la « branche », en effet, à tour de rôle sur chacun des hommes qui lui font face, en plaçant simultanément sur l'orifice de subincision et la bouche de son vis-à-vis les extrémités de la tresse, à la suite de quoi il étreint l'homme. En plaçant les deux extrémités de la ceinture aux deux extrémités ouvertes du corps de chacun des hommes successivement, il les agglutine en un même tout indivis animé par le même sentiment de vengeance. Cette conjonction des membres de l'expédition au moyen de la ceinture est complétée par le mélange de leur sang créant entre eux une intimité encore plus étroite : l'ensemble des hommes présents se font face deux à deux les uns à la suite des autres, et font saigner leur orifice de subincision au-dessus des cuisses de l'autre, et vice versa. Ce saignement mutuel est censé les lier encore plus étroitement et rendre leur trahison impossible. Ils se rassemblent ensuite et se mettent à tourner autour du monceau constitué par l'assemblage de leurs lances respectives. Ils forment dorénavant ensemble un même corps meurtrier, une seule lance indéfectible. La ceinture et le sang jouent le rôle d'adhésifs particulièrement puissants qui subsument sous l'identité du mort celles des membres de l'expédition, regroupés sous une même bannière émotionnelle, celle de la vengeance. Le partage de cet affect, si typique des phénomènes associés à la foule, résulte bien d'un processus qui vise à uniformiser les positions et les rôles initiaux des uns et des autres. Mais la constitution de ce collectif homogène se fonde sur une stricte conformité à une série d'interactions prescrites, imbriquées les unes dans les autres, et coûteuses pour ceux qui doivent s'y conformer en s'impliquant, en quelque sorte corps et âmes, sans que ne soient autorisés les débordements entre eux. De ce point de vue, cette homogénéisation s'oppose en tout point à une explosion spontanée. Elle est de fait extrêmement cadrée et c'est même la condition de l'efficience qu'on lui impute : pour que le rite ait quelque chance d'être suivi d'effet, il faut que les actions qui le sous-tendent soient contenues par l'ajustement de l'intensité émotionnelle ressentie par les uns et les autres.

On passe bien ici d’une hétérogénéité initiale des positions et des ressentis, sous-tendue par la distinction des relations de parenté qu'entretiennent le leader et ses partenaires avec le défunt, à la constitution d'un tout homogène fondée sur l'émulation émotionnelle. En effet, avant de pouvoir tuer le coupable, il faut que le justicier en chef soit à même de communiquer une soif de vengeance qui soit à la mesure de celle qu'il ressent en vertu du lien d'intimité spécifique qui l'unit au mort. On relèvera alors que la constitution de ce collectif parfaitement ajusté sur le plan affectif s'effectue par incréments successifs au travers d'une série ordonnée d'actes précis et scrupuleusement accomplis, dans lequel l'appariement, appliqué systématiquement de manière à unir physiquement chaque participant avec tous les autres, joue un rôle essentiel. C'est cet engrènement de séquences prescrites - et non le surgissement spontané d'une émotion commune - qui aboutit à la mise en scène de l'acte rituel final au cours duquel les actions des divers participants sont identiques, voire symboliquement confondues, exprimant ainsi un même sentiment de vengeance.

\section{Une symphonie concertante}

Arrivés au terme de notre présentation de quelques pratiques rituelles, nous pouvons nous interroger sur le caractère partagé des émotions qui s’y manifestent. Dans quelle mesure peut-on parler d'émotions collectives ? Répondre à cette question revient à préciser les rapports 
complexes qui existent entre, d'un côté, ce que peuvent éprouver les individus qui prennent part au rituel, et de l'autre, les états émotionnels que le rituel donne à voir.

Les moments forts des performances envisagées sont clairement organisés de manière à privilégier certains registres affectifs : la détresse ostentatoire des deuilleurs aborigènes lorsque le cercueil est fermé ou descendu dans la tombe, la fureur de ceux qui brûlent les chanteurs lors d'un gisaro, l'affabilité empressée des partenaires et la joie débridée des épisodes de « lâcher prise » dans les danses de découverte de soi. Comme le suggérait Mauss, il est plus que probable qu'il y ait au final concordance entre les ressentis personnels des divers participants et les émotions paradigmatiques exhibées par certains d'entre eux : se précipiter sur le cercueil comme pour rejoindre le mort lorsque retentissent les lamentations des pleureuses, se montrer exagérément insensible aux assauts démesurées et pathétiques des hôtes-deuilleurs, danser en manifestant comme naturellement sa "spontanéité » et son " ouverture à l'autre " en suivant ensemble les directives de l'animatrice, etc. Nos expériences d'anthropologues de terrain nous amènent à constater que cette correspondance est avant tout le résultat de l'attention qu'ils sont incités à porter sur le fait d'ajuster leur comportement à celui des autres dans le cadre d'interactions coordonnées, à la fois convenues et inhabituelles, dont l'exécution comporte une expression affective stylisée. Si l'engagement corporel dans ce type d'interactions est un impératif, leur réalisation, en revanche, n'exige en rien l'alignement des ressentis des divers participants ou la conformité de leur vécu privé avec leurs comportements publics. Dépourvus des moyens qui nous permettraient d'évaluer avec certitude le degré de similarité effective qui existe sur l'un et l'autre plan, nous préférons laisser en suspens cette question.

Toutefois, les propriétés spécifiques des actes rituels nous permettent d'avancer quelques propositions à propos des émotions rituelles. Tout d'abord, il existe à la fois une continuité et une coupure entre les manifestations quotidiennes de l'affectivité individuelle et l'expression des émotions dans les rites collectifs. La tristesse et le désarroi que donnent à voir les participants lors des cérémonies de deuil ne sont pas de nature radicalement différente des sentiments de chagrin et de détresse qu'ils peuvent manifester à d'autres occasions. Cependant, en tant qu'expression systématique et épurée de ces sentiments, médiatisée par une coordination collective et animée par des prescriptions conventionnelles, elles s'en distinguent nettement (Berthomé et Houseman 2010). Ce sont des mises en forme synthétiques et éphémères qui s'imposent à ceux qui y prennent part, non pas comme des modèles réalistes pour le comportement de tous les jours, mais comme des expériences conventionnelles, à la fois " privilégiées » (Bell 1992) et énigmatiques, à l'aune desquelles le quotidien peut être (ré)évalué. Ainsi, selon la fameuse distinction de Clifford Geertz (1993) que nous reprendrons à notre compte, les manifestations affectives de la vie courante fournissent un modèle pour l'élaboration des émotions rituelles dont l'expression offre, en retour, aux participants une modélisation de leur affectivité quotidienne.

Hors rituel, les comportements sont, en règle générale, censés être en phase avec les intentions des individus. La structuration des actes rituels semble, pour sa part, se fonder plutôt sur la reconnaissance de divergences significatives à la fois entre ce que ressentent les différents acteurs, et pour chacun d'eux, entre leurs sentiments intimes et les manifestations affectives qu'ils mettent en scène. Leurs ressentis respectifs prennent corps au travers d'actions et d'interactions conventionnellement régies par des contraintes particulièrement fortes dont l'exigence d'une coordination sans heurt. Cette régulation systémique spécialement resserrée entraîne une schématisation simplificatrice des relations rituelles qui se nouent entre les acteurs. De façon concomitante, les émotions rituelles, qui sont à comprendre comme les corrélats sensibles de ces relations ( $c f$. Bonhomme 2008), acquièrent une qualité flagrante et épurée, comme en témoignent par exemple les exhibitions dramatiques de désespoir au cours des rites funéraires, ou les démonstrations exagérées de fougue ou de tendresse dans les danses de découverte de soi. En même temps, les actions et interactions conventionnelles auxquelles se 
livrent les participants sont marquées par une complexité distinctive : le comportement des deuilleurs les identifie au défunt tout en témoignant de leur séparation radicale avec lui, les danseurs-visiteurs du gisaro s’adonnent à des lamentations pour des défunts qui ne sont pas les leurs tout en s'exhibant comme des oiseaux associés aux esprits des morts, les danseurs de découverte de soi se doivent d'entretenir des rapports à la fois excessivement intimes et sensiblement distanciés avec leurs partenaires, etc. C'est cette complexité qui rend les actes rituels, les relations qu'ils définissent, et les émotions qu'ils mettent en scène partiellement obscures et difficiles à appréhender à l'aune des intentions et attitudes propres à la vie quotidienne. Or, l'incertitude qu'introduit cette complexité, associée au caractère à la fois exacerbé et stylisé des états affectifs que les rituels donnent à voir, ne peuvent que favoriser une importante variabilité dans la façon dont chacun vit ces expériences exceptionnelles. En somme, les émotions rituelles, reconnaissables mais singularisées, interviennent davantage comme des embrayeurs de vécus affectifs sous-déterminés que comme des repères émotionnels normatifs.

Enfin, les manifestations affectives dissemblables auxquelles se livrent différentes catégories de participants se doivent d'être coordonnées entre elles. En effet, comme nous l'avons souligné au début de ce chapitre, ceux qui prennent part à un rituel élaborent leurs vécus affectifs dans le cadre d'une préoccupation commune, à savoir celle d'accomplir correctement et de concert les actes qui le constituent. Cette intention partagée des participants - un alignement de leurs concerns (Salmela 2012) focalisé sur le rite lui-même - se traduit concrètement par les obligations réciproques qui les engagent les uns vis-à-vis des autres et qui sont mises au service du bon déroulement de leur performance conjointe. Ainsi, les manifestations de tristesse lors des rites funéraires obéissent à une coordination complexe dans laquelle des personnes appartenant à des catégories distinctes se doivent de moduler leurs expressions émotionnelles respectives en fonction de celles des autres ; de même, dans les danses de découverte de soi, chacun s'efforce de rester attentif aux réactions des autres danseurs autant qu'au siennes propres en marquant cette vigilance par des gestes d'ardeur et de tendresse qui peuvent, au départ, être de convenance mais qui, au cours du déroulement de la séance, en raison d'une implication volontairement acceptée, acquièrent une efficience indéniable. Cet engagement personnel dont font preuve les participants fournit les conditions, non tant d'un partage d'une même émotion, que de l'expérience de ressentir ensemble simultanément des émotions diversifiées dont les expressions sont cependant étroitement ajustées les unes aux autres; c'est bien en ce sens, et en ce sens seulement, que les ressentis sont partagés. On retrouve dans cette mutualisation des émotions quelque chose de semblable au sentiment que Pierre Livet $(2002,135)$ appelle « la perception du partage de l'émotion » qu'il associe plus particulièrement aux fêtes rituelles (ibid. 142 ; voir aussi Kaufmann 2019 ; Quéré 2015). C'est la reconnaissance par les participants de cette émotion de partage, centrée sur l'expérience quelque peu mystérieuse du rite lui-même, qui s'exprime, par exemple, dans la détente enjouée qui règne lors de la réception qui suit un enterrement aborigène, dans les expressions de chagrin et de sympathie des danseurs-visiteurs envers leurs hôtes à la fin du gisaro, et dans les remerciements émus adressés au "groupe » qui marquent la fin de la séance de danses de découverte de soi.

Selon nous, cette mutualisation des vécus affectifs de chacun dans le cadre de l'agencement de leurs comportements prescrits respectifs se fonde sur la contribution conjointe à un accordage de l'intensité affective. Cet accordage est sous-tendu par l'intrication de la modulation gestuelle et sonore - lamentations funéraires, chants soutenus par les chœurs dans le gisaro, succession de musiques dans les danses de découverte - qui participe de sensations physiques d'ordre cénesthésique (Moisseeff 2016b) dont elle favorise l'ajustement. Ces sensations sont suscitées par la stimulation de récepteurs internes dont le fonctionnement dépend, non du système nerveux central régissant la motricité volontaire et la cognition, mais 
du système nerveux dit autonome ou neuro-végétatif qui régit des phénomènes physiologiques tels que, entre autres choses, les fréquences cardiaque et respiratoire et la sudation. Les phénomènes de cet ordre tendent à s'ajuster entre les personnes rassemblées dans un collectif. Lors d'une décharge émotionnelle particulièrement intense - événements traumatiques mais également configurations relationnelles particulièrement significatives et tendues l'amplification de ces réactions viscérales sont susceptibles de favoriser la déconnexion d'avec les afférences corticales en inhibant partiellement ou totalement la capacité à les contrôler par la volonté, l'expérience et sa mémorisation se centrant alors sur les ressentis et les perceptions associées aux circonstances qui les ont déclenchées. C'est, par exemple, ce qui est à l'origine des symptômes itératifs et particulièrement invalidants du syndrome de stress post-traumatique. Nous posons donc que, dans des situations hautement régulées mais tout aussi chargées, telles que les rituels considérés, les sensations cénesthésiques concourent en grande partie à assurer l'harmonisation de l'intensité des ressentis qui sous-tend la mutualisation des émotions.

En tant que série d'actes prescrits, un rituel ne peut pas être mal fait : soit il est reconnu comme ayant été fait comme il fallait, soit on estime qu'il n’a pas été fait du tout. Cette aptitude à induire une évaluation en termes de oui-ou-non (plutôt qu'en termes de plus-ou-moins) est un des traits qui distinguent le rituel d'autre modes performatifs comme le jeu ou le spectacle : on peut mal jouer ou mal danser sans pour autant cesser de jouer ou de danser. Mais une pratique rituelle peut néanmoins être jugée défaillante, infelicitous dirait Ronald Grimes (2010, 183), lorsque ce que nous désignons comme la mutualisation des émotions - l'émotion de partage ne se réalise pas. Par exemple, il arrive que les Aborigènes soient conviés à effectuer ensemble un rituel devant des Blancs qui ne participent qu'en tant que spectateurs au sens strict, c'est-àdire non impliqués dans son exécution. En l'absence d'obligations réciproques du même ordre entre performeurs et spectateurs, les Aborigènes se contentent de faire un spectacle. En ce cas, si les Blancs ont un ressenti, il est esthétique et/ou fondé sur la projection de ce qu'ils pensent être un rituel aborigène. En revanche, lorsque les Aborigènes participent dans le cadre de festivals aborigènes à des représentations ritualisées, parce que les uns et les autres en connaissent les enjeux et de ce fait s'y impliquent de façon comparable, il y a une véritable émulation entre eux et l'intensité émotionnelle est remarquable.

Comme nous l'avons souligné à plusieurs reprises, le bon déroulement du rite repose sur une dynamique continue de coordination des comportements des participants qui dépend en grande partie d'un ajustement réciproque de l'intensité des expressions affectives que ces comportements occasionnent. Pour ce faire, le recours à des tiers - les officiants rituels que sont, par exemple, dans les funérailles aborigènes, les pleureuses, dans le gisaro, les chanteurs, et dans les danses de découverte de soi, l'animatrice - est tout à fait crucial : ils n'agissent pas en leur nom personnel mais au nom de la charge qu'ils assument de médiatiser les relations des uns et des autres en sorte que leurs expressions affectives respectives constituent un tout cohérent. Cette intervention de tiers médiateurs va de pair avec le rôle central que joue dans ces pratiques la figure de l'appariement. La complémentarité des gestes entre les deuilleurs et chaque visiteur dans le sorry business camp aborigène sous la houlette des lamentations des pleureuses, ou l'affrontement dramatique entre visiteurs-performeurs et deuilleurs dans le gisaro, ou encore les partenariats qui se succèdent au cours des danses de découverte de soi en sont autant d'exemples tout comme l'appariement entre le justicier initiant une expédition de vengeance aranda et chacun de ses acolytes. L'interdépendance dyadique, en raison des dynamiques d'identification et de différenciation réciproques qu'elle induit, impose des contraintes particulièrement fortes aux interactions et à leur expression émotionnelle. En engendrant ainsi des sites d'intensification de l'expérience vécue des participants dont elle marque le tempo, elle s'impose comme le ressort privilégié de la structuration du champ relationnel qu'instaurent les performances rituelles. De ce point de vue, le recours à des tiers médiateurs à même d'incarner une autorité propre aux pratiques cérémonielles, d'un côté, et la 
mise en place de dispositifs d'appariement, de l'autre, contribueraient de façon décisive à l'expression rituelle des émotions.

La symphonie est caractérisée par la multiplicité des exécutants et la diversité des timbres, et le concerto par l'articulation d'un ou plusieurs instruments solistes avec l'orchestre. De ce point de vue, l'orchestration rituelle de l'expression des émotions telle que nous avons tenté de la décrire renverrait à une symphonie concertante où fusionnent les deux genres : la coordination systématique et conventionnellement réglée des corps d'un ensemble disparate de participants médiatise la constitution d'un collectif extra-ordinaire, à la fois en continuité avec et disjoint de la vie quotidienne, au sein duquel, sans abolir la distinction de leurs places respectives, l'accordage du vécu affectif idiosyncrasique des individus particuliers à celui des autres concourt à la réalisation d'une expérience émotionnelle partagée d'une ampleur exceptionnelle.

Pour Claude Lévi-Strauss (1962, 3-47), l'œuvre créée par un artiste doit être appréhendée comme le modèle réduit d'un phénomène permettant au spectateur de saisir celuici, non plus sous l'angle de ses parties séparées, mais dans sa globalité, expérience sur laquelle se fonde, selon cet auteur, la spécificité du plaisir esthétique. Dans cette optique, « [...] la vertu intrinsèque du modèle réduit est qu'il compense la renonciation à des dimensions sensibles par l'acquisition de dimensions intelligibles » (ibid., 36). Pour notre part, nous suggérons que si le rite est bien une création culturelle dont découle une expérience esthétique, c'est-à-dire sensible et affective comme l'entend Jean-Louis Génart dans ce volume, participant de l'appréciation de la réussite d'un rite, et s'il peut effectivement être considéré comme un petit monde synthétique, en dehors du cas où il est érigé en spectacle pour touristes, on ne peut parler de spectateurs dans le même sens que celui dont use Lévi-Strauss pour qualifier l'admirateur d'une œuvre d'art. Dans le rituel, l'ensemble des participants contribuent activement à la production de l'expérience globale par les obligations réciproques qui les engagent les uns vis-à-vis des autres. Nous inverserons donc la proposition lévi-straussienne concernant la valeur intrinsèque du modèle réduit qu'il applique à l'œuvre d'art pour suggérer, en conclusion, que la vertu intrinsèque du rituel est qu'il compense la renonciation à des dimensions intelligibles par l'acquisition de dimensions sensibles.

\section{BIBLIOGRAPHIE}

Allard Olivier, (2013), « To Cry one's Distress: Death, Emotion, and Ethics among the Warao of the Orinoco Delta », Journal of the Royal Anthropological Institute 19 : 545-561.

Altglas Véronique, (2014), From Yoga to Kabbalah. Religious Exoticism and the logics of bricolage, Oxford / New York, Oxford University Press.

Bachollet Marie-Sophie et Daniel Marcelli, (2010), « Le dialogue tonico-émotionnel et ses développements », Enfances \& Psy 49 : 14-19.

Bell Catherine, (1992), Ritual Theory, Ritual Practice, Oxford / New York, Oxford University Press.

Berndt Ronald et Catherine Berndt, (1945), « A Preliminary Report of Fieldwork in the Ooldea Region, Western South Australia », Oceania XV (3) : 239-75.

Berthomé François et Michael Houseman, (2010), « Ritual and Emotions: Moving Relations, Patterned Effusions », Religion and Society 1 (1) : 57-75. 
Bonhomme Julien (2008), « Des pleurs ou des coups. Affects et relations dans l'initiation au Bwete Misoko (Gabon) », Systèmes de pensée en Afrique Noire, p. 133-163. En ligne: [https://journals.openedition.org/span/785].

Bourdieu Pierre, (1979), La distinction. Critique sociale du jugement, Paris, Editions de Minuit.

Collins Randall, (2004), Interaction Ritual Chains, Princeton, Princeton University Press.

Durkheim Emile. 1913. Les Formes élémentaires de la vie religieuse : le système totémique en Australie, Paris, Presses Universitaires de France.

Feld Steven, (1982), Sound and Sentiment. Birds, Weeping, Poetics and Song in Kaluli Expression, Philadelphia, University of Pennsylvania Press.

Fingerman Karen L., (2009), « Consequential Strangers and Periferal Partners: The Importance of Unimportant Relationships », Journal of Family Theory and Review 1 : 69-86.

Geertz Clifford, (1973), "Religion as a Cultural System », in Geertz Clifford (dir.), The Interpretation of Cultures: Selected Essays, New York, Basic Books, 87-125.

Giddens Anthony, (1991), Modernity and Self-Identity. Self and Society in the Late Modern Age, Cambridge, Blackwell.

Glaskin Katie, Myrna Tonkinson, Yasmine Musharbach et Victoria Burbank, (2008), Mortality, Mourning and Mortuary Practices in Indigenous Australia, Farnham / Burlington, Ashgate Publishing.

Gratier Maya, (2001), « Harmonies entre mère et bébé. Accordage et contretemps », Enfances \& Psy $13: 9-15$.

Grimes, Ronald (2010 [1990]), Ritual Criticism: Case Studies in its Practice, Essays on its Theory, 2ème édition, Waterloo, Ritual Studies International.

Houseman Michael, (2012), Le rouge e(s)t le noir. Essais sur le rituel, Toulouse, Presses Universitaires du Mirail.

Houseman Michael, (2016), « Comment comprendre l'esthétique affectée des cérémonies New Age et néopaïennes ? » Archives de Sciences Sociales des Religions 174, p. 213-237.

Houseman Michael, (2020), « Becoming autonomous together: distanced intimacy in dances of self-development », in Salomonsen Jone, Michael Houseman, Sarah Pike et Graham Harvey (dir.), Reassembling Democracy. Ritual as Cultural Resource, London, Bloomsbury Publishing, 87-104.

Houseman Michael et Marie Mazzella di Bosco, (2020), « Dances of self-development as a resource for participatory democracy », in Pike Sarah M., June Salomonsen et Paul-François Tremlett (dir.), Ritual and Democracy: Protests, Publics, and Performances, Sheffield, Equinox Publishing, 115-138.

Houseman Michael, Marie Mazzella di Bosco et Emmanuel Thibault, (2016), " Renaître à soimême. Pratiques de danses rituelles en Occident contemporain », Terrain 66 : 62-85.

Humphrey Caroline et James Laidlaw, (1994), The Archetypal Actions of Ritual. A Theory of Ritual Illustrated by the Jain Rite of Worship, Oxford, Clarendon Press.

Kaufmann, Laurence (2019), « Emotions collectives », in Gloria Origgi (ed.), Dictionnaire des passions sociales, Paris, Presses Universitaires de France, p. 191-199.

Le Bon Gustave, (1905), Psychologie des foules, 9e édition, Paris, Édition Félix Alcan [1895]. Lévi-Strauss, Claude, (1962), La pensée sauvage, Paris, Plon. 
Livet, Pierre (2002), Émotions et rationalité morale, Paris, Presses Universitaires de France. Malinowski Bronislaw, (1927), Sex and Repression in Savage Society, New York, Kegan Paul. Mauss Marcel, (1921), «L'expression obligatoire des sentiments (rituels oraux funéraires australiens) », Journal de psychologie 18 : 425-34.

Mazzella di Bosco Marie, (sous presse), « 'Danser la relation'. Interactions en mouvement dans les danses libres en conscience », Ateliers d'anthropologie 47.

Moisseeff Marika, (1994), «Les objets cultuels aborigènes ou comment représenter l’irreprésentable », Genèses 17 : 4-32.

Moisseeff Marika, (1995), Un long chemin semé d'objets cultuels : le cycle initiatique aranda. Paris, EHESS.

Moisseeff Marika, (2012), «L'Objet de la transmission : un choix culturel entre sexe et reproduction ", in Schwering Karl-Leo (dir.), Se construire comme sujet entre filiation et sexuation, Toulouse, Erès, 47-76.

Moisseeff Marika, (2016a), «Le mort, ses proches et les autres : ici et ailleurs », in Caiozzo Anna, (dir.), Mythes, rites et émotions, les funérailles le long de la Route de la soie, Paris, Honoré Champion, 29-48.

Moisseeff Marika, (2016b), "Cadavres et Churinga : des objets cultuels exemplaires ? », Arch. de Sc. soc. des Religions 174 : 255-278.

Moisseeff Marika, (2017), "Setting Free the Son, Setting Free the Widow: Relational Transformation in Arrernte Life-Cycle Rituals (Central Australia) ", Anthropological Forum 27 (1) : 34-48.

Moisseeff Marika, (2020), « Dealing with Death in Contemporary Western Culture: a View from afar » in Salomonsen Jone, Michael Houseman, Sarah Pike et Graham Harvey (dir.), Reassembling Democracy. Ritual as Cultural Resource, London, Bloomsbury Publishing, 179191.

Munn Nancy, (1995), "An Essay on the Symbolic Construction of Memory in the Kaluli Gisalo », in Coppet de Daniel et André Iteanu (dir.), Cosmos and Society in Oceania, Oxford \& Herndon, 83-104.

Quéré Louis, (2015), « Nature et formes de l'émotion collective », CEMS Occasional Paper, 32, Paris, EHESS. http://cems.ehess.fr/docannexe/file/3689/op32_louis_quere.pdf

Radcliffe-Brown Alfred Reginald, (1922), The Andaman islanders. A study in social anthropology, Cambridge, Cambridge University Press.

Salmela, Mikko (2012), « Shared Emotions », Philosophical Explorations, 15 (1), p. 33-46.

Schieffelin Edward, (1976), The Sorrow of the Lonely and the Burning of the Dancers, New York, Saint Martin’s Press.

Spencer Baldwin et Francis Gillen, (1927), The Arunta. A Study of a Stone-Age People, Londres, Macmillan.

Stern Daniel, (1985), The Interpersonal World of the Infant. A View from Psychoanalysis and Developmental Psychology, New York, Basic Books.

Tonkinson Myra, (2008), « Solidarity in Shared Loss : Death-related Observances among the Martu of the Western Desert », in Glaskin Katie et al. (dir.), Mortality, Mourning and Mortuary Practices in Indigenous Australia, Farnham / Burlington, Ashgate Publishing, 37-53. 
Turner Victor. (1969), The Ritual Process: Structure and Anti-Structure, Chicago, Aline Publishing.

Van Gennep Arnold, (1981 [1909]), Les Rites de passage, Paris, Picard [1909]

Whitehouse Harvey, 2000, Arguments and Icons: Divergent Modes of Religiosity, Oxford, Oxford University Press. 